\title{
Upconversion-based lidar measurements of atmospheric $\mathrm{CO} 2$
}

\author{
Høgstedt, Lasse; Fix, Andreas; Wirth, Martin; Pedersen, Christian; Tidemand-Lichtenberg, Peter
}

Published in:

Optics Express

Link to article, DOI:

10.1364/OE.24.005152

Publication date:

2016

Document Version

Publisher's PDF, also known as Version of record

Link back to DTU Orbit

Citation (APA):

Høgstedt, L., Fix, A., Wirth, M., Pedersen, C., \& Tidemand-Lichtenberg, P. (2016). Upconversion-based lidar measurements of atmospheric CO2. Optics Express, 24(5), 5152-5161. https://doi.org/10.1364/OE.24.005152

\section{General rights}

Copyright and moral rights for the publications made accessible in the public portal are retained by the authors and/or other copyright owners and it is a condition of accessing publications that users recognise and abide by the legal requirements associated with these rights.

- Users may download and print one copy of any publication from the public portal for the purpose of private study or research

- You may not further distribute the material or use it for any profit-making activity or commercial gain

- You may freely distribute the URL identifying the publication in the public portal

If you believe that this document breaches copyright please contact us providing details, and we will remove access to the work immediately and investigate your claim. 


\title{
Upconversion-based lidar measurements of atmospheric $\mathrm{CO}_{2}$
}

\author{
Lasse Høgstedt, ${ }^{1, *}$ Andreas Fix, ${ }^{2}$ Martin Wirth, ${ }^{2}$ Christian Pedersen, ${ }^{1}$ \\ and Peter Tidemand-Lichtenberg ${ }^{1}$ \\ ${ }^{1}$ Technical University of Denmark, Department of Photonics Engineering, 4000 Roskilde, \\ Denmark \\ ${ }^{2}$ DLR(German Aerospace Center), Institute of Atmospheric Physics, 82234 Oberpfaffenhofen, \\ Germany \\ *lhog@fotonik.dtu.dk
}

\begin{abstract}
For the first time an upconversion based detection scheme is demonstrated for lidar measurements of atmospheric $\mathrm{CO}_{2}$-concentrations, with a hard target at a range of $3 \mathrm{~km}$ and atmospheric backscatter from a range of $\sim 450 \mathrm{~m}$. The pulsed signals at $1572 \mathrm{~nm}$ are upconverted to $635 \mathrm{~nm}$, and detected by a photomultiplier tube, to test how the upconversion technology performs in a long range detection system. The upconversion approach is compared to an existing direct detection scheme using a near-IR detector with respect to signal-to-noise ratio and quantum efficiency. It is for the first time analyzed how the field-of-view of a receiver system, for long range detection, depends critically on the parameters for the nonlinear upconversion process, and how to optimize these parameters in future systems.
\end{abstract}

(C) 2016 Optical Society of America

OCIS codes: (190.7220) Upconversion; (280.1910) DIAL, differential absorption lidar; (040.3060) Infrared; (010.0280) Remote sensing and sensors.

\section{References and links}

1. P. Ciais, C. Sabine, G. Bala, L. Bopp, V. Brovkin, J. Canadell, A. Chhabra, R. DeFries, J. Galloway, M. Heimann, C. Jones, C. Le Quéré, R. B. Myneni, S. Piao, and P. Thornton, "2013: Carbon and Other Biogeochemical Cycles," in Climate Change 2013: The Physical Science Basis. Contribution of Working Group I to the Fifth Assessment Report of the Intergovernmental Panel on Climate Change, Stocker, T.F., D. Qin, G.-K. Plattner, M. Tignor, S.K. Allen, J. Boschung, A. Nauels, Y. Xia, V. Bex and P.M. Midgley (eds.) (Cambridge University, 2013).

2. G. Ehret, C. Kiemle, M. Wirth, A. Amediek, A. Fix, and S. Houweling, "Space-borne remote sensing of $\mathrm{CO}_{2}$, $\mathrm{CH}_{4}$, and $\mathrm{N}_{2} \mathrm{O}$ by integrated path differential absorption lidar: a sensitivity analysis," Appl. Phys. B 90, 593-608 (2008).

3. A. Fix, A. Amediek, C. Büdenbender, G. Ehret, M.Quatrevalet, M. Wirth, J. Löhring, R. Kasemann, J. Klein, H.-D. Hoffmann, and V. Klein, "Development and First Results of a new Near-IR Airborne Greenhouse Gas Lidar," in Advanced Solid State Lasers, OSA Technical Digest (online) (Optical Society of America, 2015), paper ATh1A.2.

4. D. Sakaizawa, C. Nagasawa, T. Nagai, M. Abo, Y. Shibata, M. Nakazato, and T. Sakai, "Development of a 1.6 $\mu \mathrm{m}$ differential absorption lidar with a quasi-phase-matching optical parametric oscillator and photon-counting detector for the vertical $\mathrm{CO}_{2}$ profile," Appl. Opt. 48, 748-757 (2009).

5. A. Rogalski, Infrared Detectors, 2nd ed. (CRC Press, 2011).

6. H. Xi, G. Shentu, M. Shangguan, X. Xi, X. Ji, C. Wang, J. Zhang, J. S. Pelc,M. M. Fejer, Q. Zhang, X. Dou, and J. Pan, "Long-range micro-pulse aerosol lidar at $1.5 \mu \mathrm{m}$ with an upconversion single-photon detector," Opt. Lett. 40, 1579-1582 (2015).

7. T. Wong, J. Yu, Y. Bai, W. Johnson, S. Chen, M. Petros, and U. N. Singh, "Sensitive infrared signal detection by upconversion technique," Opt. Eng. 53, 107102 (2014). 
8. V. G. Dmitriev, G. G. Gurzadyan, and D. N. Nikogosyan, Handbook of Nonlinear Optical Crystals, 2nd ed. (Springer, 1995).

9. J. Löhring, J. Luttmann, R. Kasemann, M. Schlösser, J. Klein, H. Hoffmann, A. Amediek, C. Büdenbender, A. Fix, M. Wirth, M. Quatrevalet, and G. Ehret, "INNOSLAB-based single-frequency MOPA for airborne lidar detection of CO2 and methane," Proc. SPIE 8959, 89590J (2014).

10. L. Høgstedt, P. Tidemand-Lichtenberg, and C. Pedersen "Design of a solid state laser for low noise upconversion detection of near infrared light," in Advanced Solid State Lasers, OSA Technical Digest (online) (Optical Society of America, 2015), paper AM5A.6.

11. L. Høgstedt, J. S. Dam, A. Sahlberg, Z. Li, M. Aldén, C. Pedersen, and P. Tidemand-Lichtenberg, "Low-noise mid-IR upconversion detector for improved IR-degenerate four-wave mixing gas sensing," Opt. Lett. 39, 53215324 (2014).

12. J. S. Dam, P. Tidemand-Lichtenberg, and C. Pedersen, "Room-temperature mid-infrared single-photon spectral imaging," Nat. Photonics 6, 788-793 (2012).

13. L. S. Rothman, I. E. Gordon, Y. Babikov, A. Barbe, D. Chris Benner, P. F. Bernath, M. Birk, L. Bizzocchi, V. Boudon, L. R. Brown, A. Campargue, K. Chance, E.A. Cohen, L. H. Coudert, V. M. Devi, B. J. Drouin, A. Fayt, J.-M. Flaud, R. R. Gamache, J. J. Harrison, J.-M. Hartmann, C. Hill, J. T. Hodges, D. Jacquemart, A. Jolly, J. Lamouroux, R. J. Le Roy, G. Li, D. A. Long, O. M. Lyulin, C. J. Mackie, S. T. Massie, S. Mikhailenko, H. S. P. Mller, O. V. Naumenko, A. V. Nikitin, J. Orphal, V. Perevalov, A. Perrin, E. R. Polovtseva, C. Richard, M. A. H. Smith, E. Starikova, K. Sung, S. Tashkun, J. Tennyson, G. C. Toon, V. G. Tyuterev, and G. Wagner, "The HITRAN2012 molecular spectroscopic database,” J. Quant. Spectrosc. Radiat. Transf. 130, 4-50 (2013)

14. J. S. Pelc, L. Ma, C. R. Phillips, Q. Zhang, C. Langrock, O. Slattery, X. Tang, and M. M. Fejer, "Long-wavelengthpumped upconversion single-photon detector at $1550 \mathrm{~nm}$ : Performance and noise analysis," Opt. Express 19, 21445-21456 (2011).

15. C.L. Tang, "Spontaneous emission in the frequency up-conversion process in nonlinear optics," Phys. Rev. 182, 367-374 (1969).

16. J. Chang, Z. Yang and Q. Sun, "Broadband mid-infrared difference frequency generation in uniform grating periodically poled lithium niobate," Optik 126, 1123-1127 (2015).

17. A. Bostani, M. Ahlawat, A. Tehranchi, R. Morandotti, and R. Kashyap, "Design, fabrication and characterization of a specially apodized chirped grating for reciprocal second harmonic generation," Opt. Express 23, 5183-5189 (2015).

18. X. Sanchez-Lozano and J. L. Lucio, "Spontaneous parametric down conversion in chirped, aperiodically-poled crystals," Int. J. Quantum Inf. 13, 1550032 (2015).

\section{Introduction}

For a prediction of the rate of climate change during the $21^{\text {st }}$ century, there is an urgent need to better understand the global carbon cycle. This concerns, in particular, the understanding of the processes that control how the most important anthropogenic greenhouse gases, $\mathrm{CO}_{2}$ and $\mathrm{CH}_{4}$, flow between the various reservoirs [1].

Currently, there is a significant lack in accurate observations from regional to global scales, needed to infer the fluxes of these important greenhouse gases. A technique that shows potential to provide highly accurate measurements e.g. from ground, aircraft or satellites is the Differential Absorption Lidar (DIAL) technique or its modification, the Integrated Path DIAL (IPDA) $[2,3]$, applied in the near infrared spectral range. The IPDA technique has proven viable for airborne measurements of integrated path concentrations [3], but a pulsed DIAL system is required to get vertical concentration profiles. This has been demonstrated earlier at $1.6 \mu \mathrm{m}$ with a near infrared PMT and 5 hours of integration time [4], but much shorter integration times are needed for airborne measurements. The limiting factor to obtain this is the noise properties of the receiver system. There can be four orders of magnitude improvement in detectivity [5] by changing the detection system from an InGaAs PIN-diode to a visible light photo multiplier tube (VIS-PMT) and it is thus an advantage to shift the signal wavelength, from the near infrared to the visible spectral range.

In this work we demonstrate, what we believe, is the first lidar measurement of $\mathrm{CO}_{2}$ using an upconversion module together with a photomultiplier tube (PMT) to detect the $1572 \mathrm{~nm}$ signal at $635 \mathrm{~nm}$. Upconversion is a nonlinear parametric process that provide extra energy to 
the infrared signal photons by the following energy conservation relation

$$
\frac{1}{\lambda_{\mathrm{VIS}}}=\frac{1}{\lambda_{\mathrm{CO}_{2}}}+\frac{1}{\lambda_{\mathrm{Mix}}}
$$

In the scope of this investigation $\lambda_{\mathrm{VIS}}=635 \mathrm{~nm}, \lambda_{\mathrm{Mix}}=1064 \mathrm{~nm}$ and $\lambda_{\mathrm{CO}_{2}}=1572 \mathrm{~nm}$. This shift the near infrared photons to the visible region where efficient low noise detectors exist.

We will analyse the advantages and challenges with signal upconversion and discuss how this technology has the potential to improve the sensitivity of $\mathrm{CO}_{2}$ measurements by orders of magnitude. With an improved sensitivity it will be possible to gather the vertical $\mathrm{CO}_{2}$ concentration profile and get an extra dimension in the global mapping of the atmospheric greenhouse gas concentrations.

An upconversion based system for long range detection has been demonstrated before for detection of atmospheric aerosols [6]. At a signal wavelength of $1548 \mathrm{~nm}$ a $50 \mathrm{~mm}$ long periodically poled lithium niobate (PPLN) waveguide was used in a long-wavelength pumped single pass experiment combined with a SI-APD. For the potential use with a $\mathrm{CO}_{2}$ lidar a high efficiency $2.055 \mu \mathrm{m}$ intracavity upconversion system shifting the signal to $0.71 \mu \mathrm{m}$ has been demonstrated in the lab using a $50 \mathrm{~mm}$ bulk PPLN crystal [7].

In this paper we demonstrate how an intracavity upconversion system is applied together with a VIS-PMT as the receiver in a pulsed DIAL system for detection of atmospheric $\mathrm{CO}_{2}$. We measure the on-line and off-line return signals backscattered from a hard target, which in our case is a forest at a distance of $\sim 3 \mathrm{~km}$, and from atmospheric aerosol at a distance of $\sim 450 \mathrm{~m}$. These measurements give rise to, what we believe, is the first analysis of the inherent angular dependency, as an important design parameter for upconversion based long range detection systems.

\section{Theoretical considerations}

In efficient long range detection systems the overlap between the field-of-view (FOV) for the transmitted beam and the receiver system are matched. The FOV is defined as the full divergence or acceptance angle for the transmitter and receiver respectively. The conservation of etendue or the angle-area-product is a key relation in the design of the optical receiver. In a long range system the area of the collecting telescope is typically much larger than the area of the detector. When the signal is downscaled, to match the detector, the incident angle increase and it is not unusual with angles close $90^{\circ}$. This works well with the approximately linear angle dependency for conventional detectors, but with an upconversion based receiver system the angular acceptance is strictly limited by the nonlinear parameteric process. This requires a different design of the scaling optics and the the FOV may be limited by the upconversion process as displayed in the inset in Fig. 1, where a conceptual sketch of the DIAL system is displayed.

The efficiency of the nonlinear upconversion process is given by Eq. (2) [8] assuming plane waves, no pump depletion and perfect overlap of the interacting fields.

$$
\eta_{\text {up }}=\frac{P_{635}}{P_{1572}}=\frac{8 d_{\mathrm{eff}}^{2} \pi^{2} P_{1064}}{c n_{1064} n_{635} n_{1572} \lambda_{1572}^{2} \varepsilon_{0} A} L^{2} \frac{\sin ^{2}\left(\frac{\Delta k L}{2}\right)}{\left(\frac{\Delta k L}{2}\right)^{2}}
$$

Where $d_{\text {eff }}$ is the effective nonlinearity of the PPLN crystal, $P_{i}$ the specific power, $c$ the speed of light, $n_{i}$ the wavelength-specific and temperature dependent index of refraction, $\lambda_{1572}$ the signal wavelength, $\varepsilon_{0}$ the vacuum permittivity, $A$ is the effective overlap area in the crystal between the pump laser and the incoming signal, $L$ the crystal length and $\Delta k$ the phasemismatch of the parametric process. Eq. 2 is seen to depend linearly on the pump power and quadratic on the crystal length if the upconversion process is phasematched, i.e. $\Delta k=0$. The phasemismatch is 


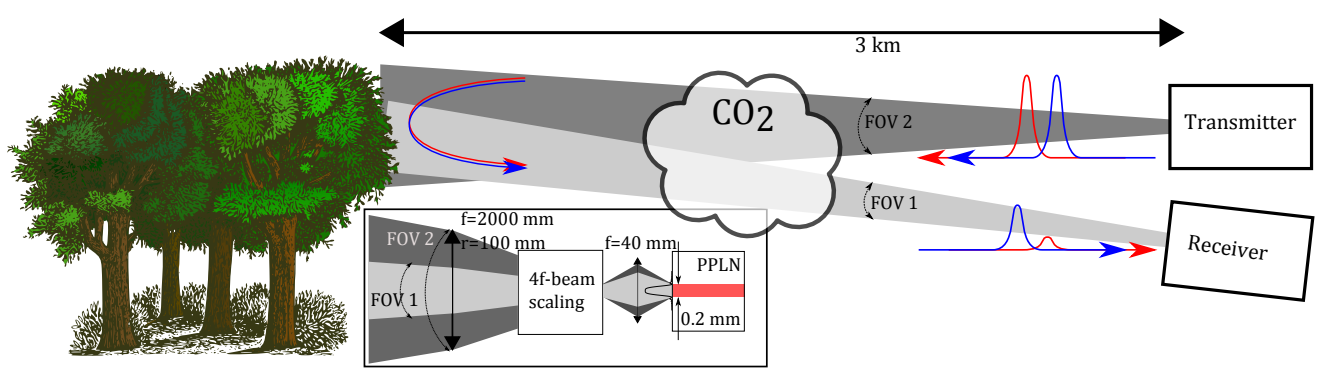

Fig. 1. Conceptual sketch of the DIAL measurement setup. The inset shows how the upconversion process defines the effective field-of-view for the receiver system.

calculated from Eq. (3) where $\Lambda_{\text {PPLN }}$ is period of the poling structure in the lithium niobate crystal.

$$
\Delta k=k_{1064}-k_{635} \cos \left[\arcsin \left(\frac{k_{1572}}{k_{635}} \sin (\theta)\right)\right]+k_{1572} \cos (\theta)+\frac{2 \pi}{\Lambda_{\mathrm{PPLN}}}, k_{i}=\frac{2 \pi}{\lambda_{i}} n\left(\lambda_{i}, T\right)
$$

Together, Eqs. (2) and (3) show how the upconversion efficiency depends on the angle, $\theta$, of the incoming light. Assuming a constant temperature, optimized for collinear phasematch, it follows that the efficiency will drop for non-collinear angles and that this defines the angular acceptance bandwidth of the upconversion process. The acceptance bandwidth is specific for the choice of wavelength and decreases for increasing crystal length. PPLN is chosen as the nonlinear material due to the high effective nonlinearity and the large potential for parameter engineering.

In addition to the efficiency of the nonlinear process, the overall efficiency of an upconversion based receiver system, $\eta_{\text {total }}$, will depend on the efficiency of the visible detector $\eta_{\text {vis.det. }}$ and the overlap between the mixing laser and the signal inside the nonlinear crystal, $\eta_{\text {overlap }}$,

$$
\eta_{\text {total }}=\eta_{\text {overlap }} \eta_{\text {up }} \eta_{\text {vis.det. }}
$$

where $\eta_{\text {overlap }}$ is calculated as the relation between the signal and mixing laser area. To describe the efficiency when applied for a long range receiver system the angular distribution of the power should to be included in $\eta_{\text {up }}$. This can be described with a weighted quantum efficiency, defined as

$$
Q E_{\text {weighted }}=\int_{0}^{\pi} \eta_{\mathrm{up}}(\theta) \frac{\lambda_{635}}{\lambda_{1572}} \Gamma(\theta) 2 \pi \theta \mathrm{d} \theta
$$

where $\Gamma(\theta)$ is the angular distribution of the incoming power. Together with the conservation of etendue, Eqs. (4) and (5) form the basis for the optimization of an upconversion based receiver system.

With a $20 \mathrm{~mm}$ long PPLN crystal and a pump beam with a diameter of $100 \mu \mathrm{m}$, the full width half maximum (FWHM) of the external acceptance angle for the upconversion process is $1.8^{\circ}$. The small acceptance angle together with the small overlap area in the crystal will constrain the design of the rest of the long range detection system and complicate a direct replacement of the InGaAs detector with the upconversion detector. A larger overlap area in the crystal results in a lower conversion efficiency for the collinear part as given by Eqs. (2) and (3). This can be compensated by a longer crystal, but at the expense of a lower angular acceptance bandwidth. It is, however, possible to find a compromise between the etendue and the QE that justifies the shift of wavelength to the visible regime and this will be discussed in further details in section 4.4. 


\section{Experimental details}

The upconversion detector system is tested using the airborne demonstrator for the French/German MERLIN mission [3] developed at DLR. For the experiments described in the following, the upconversion detection system was set-up side by side to this lidar instrument to allow for a comparison between the direct detection (using InGaAs PIN diodes) and the upconversion approach. An overview of the transmitter and receiver of the airborne demonstrator is found in Fig. 2 together with the realization of the upconversion based test receiver setup. The MERLIN demonstrator is based on two optical parametric oscillators (OPOs) which are

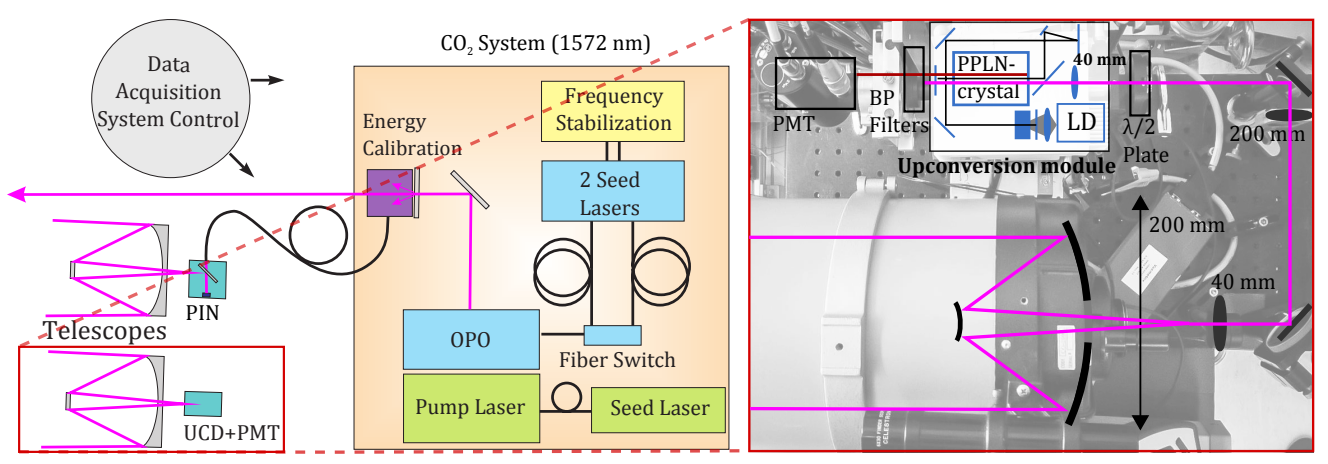

Fig. 2. Left: Experimental details of the lidar transmitter system with the original receiver system side by side with the upconversion test setup. All IPDA measurements were controlled by the common data acquisition system. Right: Image of the upconversion detection system with diagram overlay. NIR signal $\rightarrow$ Scaling and guiding $\rightarrow$ Intracavity upconversion $\rightarrow$ Visible PMT.

diode-pumped by means of injection seeded, Q-switched Nd:YAG lasers in a master-oscillator power-amplifier configuration. Originally, the system generates radiation at both $1572 \mathrm{~nm}$ and $1645 \mathrm{~nm}$, dedicated to $\mathrm{CO}_{2}$ and methane, respectively. In the context of this work only the $\mathrm{CO}_{2}$ part was employed. The IPDA technique requires the generation of two wavelengths that act as the on- and off-line signals and this is achieved by injection seeding from two stabilized distributed feedback lasers. The system operates in a double pulse mode at $50 \mathrm{~Hz}$ and the on- and off-line pulses are emitted with a pulse length of $20 \mathrm{~ns}$ and a time separation of $500 \mu \mathrm{s}$. For the experiments described the on-line and off-line wavelength were chosen at $1572.0214 \mathrm{~nm}$ and $1572.1241 \mathrm{~nm}$, respectively. The single pulse energy exceeded $10 \mathrm{~mJ} /$ pulse yielding an average power of $\sim 1 \mathrm{~W}$. The divergence angle of the transmitted beam was $\sim 3 \mathrm{mrad}$. The receiver system in the demonstrator used for comparison uses a $200 \mathrm{~mm}$ diameter telescope and a $1 \mathrm{~mm}$ InGaAs PIN detector. Further details on the MERLIN demonstrator is found in [3] and for the laser system specifically see [9].

The upconversion based receiver system consists of four main parts: An 8" Cassegrain telescope, beam scaling and guiding optics, the upconversion module, filters and the visible PMT. The telescope has adjustable focus, used for optimization of the signal image in the PPLN crystal. The beam guiding and scaling ensures optimal overlap between the pump and the signal in the PPLN crystal according to the etendue conservation law. An $8 \mathrm{f}$ scaling was chosen to give an effective magnification of 250. From the dimensions of the crystal a signal with a $800 \mu \mathrm{m}$ image, in the crystal, and incident angles up to $\pm 110 \mathrm{mrad}$ is the largest that can pass through the upconversion channel. This translates into an effective FOV close to $1 \mathrm{mrad}$ for the upconversion receiver system. Before the signal enters the upconversion module it passes through a half-wave plate that turns the polarization to match the polarization of the intracavity pump beam, in order to maximize the upconversion efficiency. 
The upconversion module are built as a compact, robust and portable unit consisting of a $20 \mathrm{~mm}$ PPLN crystal placed in a $1064 \mathrm{~nm}$ cavity, to achieve high mixing power. The high finesse mixing laser cavity shown in Fig. 2 is build with high reflectivity mirrors ( $\mathrm{R}>99.9 \%$ at $1064 \mathrm{~nm}$ ). For these measurements it typically operates with an intracavity power at $50 \mathrm{~W}$, calculated using a calibrated output mirror. The cavity is designed to have a clear path through the PPLN crystal for an incoming signal, while having two beam waists. $w_{\text {PPLN }}=100 \mu \mathrm{m}$ in the PPLN crystal and $w_{\mathrm{ND}: \mathrm{YVO}_{4}}=280 \mu \mathrm{m}$ in the $5 \mathrm{~mm}$ long $0.5 \% \mathrm{Nd}: \mathrm{YVO}_{4}$ laser crystal. The PPLN crystal is poled by Covesion in five $1 \mathrm{~mm} \times 1 \mathrm{~mm}$ channels with periods from $11.8 \mu \mathrm{m}$ $15.8 \mu \mathrm{m}$. The $11.8 \mu \mathrm{m}$ is applied for the upconversion of $1572 \mathrm{~nm}$ to $635 \mathrm{~nm}$. To control the phasematch of the upconversion process, the PPLN crystal is placed in an oven and the temperature is adjusted to maximize the upconversion efficiency. With a signal at $1572 \mathrm{~nm}$ the optimal crystal temperature is measured to $122^{\circ} \mathrm{C}$, and the wavelength dependent phasematch acceptance here is wide enough to cover both the on and off resonance wavelength simultaneously. After the upconversion module a VIS-PMT (Hamamatsu R928) is placed behind two OD4 band pass filters with a $10 \mathrm{~nm}$ window, centered at $632 \mathrm{~nm}$.

The noise properties and efficiency of the upconversion module is described in [10] and a similar setup using a PPLN crystal with a different poling period has been applied both for low noise point detection and image upconversion of mid-infrared light $[11,12]$.

\section{Results and discussion}

Long range $\mathrm{CO}_{2}$ detection is demonstrated experimentally, and the different noise contributions are discussed together with an investigation of the practical challenges in the angular acceptance for the upconversion process.

\subsection{Atmospheric $\mathrm{CO}_{2}$ measurements}

A forest rising on a hill $3 \mathrm{~km}$ away was used as a hard target for the IPDA measurements of $\mathrm{CO}_{2}$ and the on- and off-resonance data are shown in Fig. 3(a), both for the combined upconversionPMT detector, and for the detection with the InGaAs diode. This demonstrates the first upconversion based IPDA measurement, where the difference between on- and off-resonance signals originates from atmospheric $\mathrm{CO}_{2}$ in the beam path. The off-resonance peaks are calibrated with respect to the energy reference and normalized to one. The inset shows a comparison of the noise away from the signal peak, giving a ratio of 3.7 between the standard deviations in the two detection systems. This translates into a comparison of the SNR and show that the MERLIN demonstrator is still around 4 times better than the current configuration of the upconversion based system.

The $30 \%$ reduction for the on-resonance signal complies with the expected value for a $6 \mathrm{~km}$ path in US standard atmosphere at $20^{\circ} \mathrm{C}$ using the HITRAN2012 [13] spectral line database. The offset of the pulses received by the two detectors is a result of electronic delay difference in the acquisition systems and the different response times for the detectors.

Range resolved $\mathrm{CO}_{2}$ detection from atmospheric backscattering on a $450 \mathrm{~m}$ distance is demonstrated in Fig. 3(b), that shows the received signal from a telescope alignment optimized for a signal backscattered close to the transmitter. The intensities in the plot are calibrated to the energy reference peak, first in the plot, but still have arbitrary units and cannot be used for a direct comparison of the different detection methods. It is, however, clear that both methods detect $\mathrm{CO}_{2}$ in the atmosphere and that an extension of the detection distance requires an improved SNR. It was expected that the PMT signal resembled the InGaAs proximity signal, but due to the angle limitations and the larger separation between the transmitter and the receiver, for the upconversion system, the overlap occurs at larger distances, as seen in the figure. 

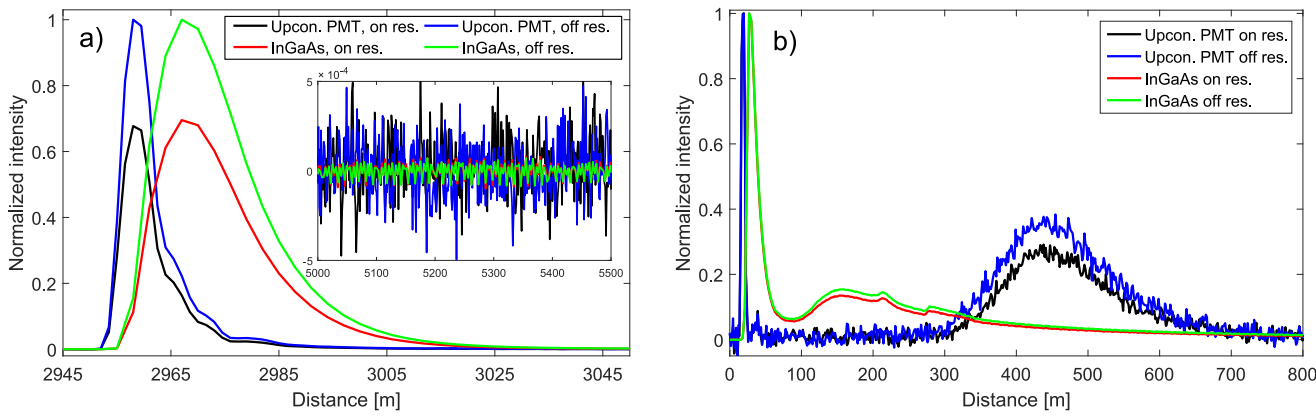

Fig. 3. a) Backscattered signal from forest on a hill $3 \mathrm{~km}$ away from the laboratory. Measured on/off resonance at the $1572 \mathrm{~nm} \mathrm{CO}_{2}$ spectral line. b) Signal from a different alignment configuration optimized for atmospheric backscattered signal. Both plots are averaged over 5 minutes.

\subsection{Noise contributions}

DIAL measurements have noise contributions from both atmospheric scattering of sunlight and the inherent dark noise in the detector. This is also the case with the combined upconversion module and PMT system, but there are three important differences.

- Due to the change in wavelength from infrared light to visible light the inherent detector noise will be lower in the case with the visible PMT compared to the InGaAs detector. This improvement can be as much as four orders of magnitude [5].

- The limited acceptance bandwidth from the phasematch condition in the upconversion process function as a $<1 \mathrm{~nm}$ bandpass filter. This, in combination with conventional bandpass filters after the upconversion module; give a very low background level. With a bandstop filter at $632 \mathrm{~nm}$ before the upconversion, inverse of the bandpass filter after the upconversion, the atmospheric noise contribution can be reduced further.

- Different noise sources are inherent for the upconversion process: A low amount of upconverted thermal photons [12], upconverted Raman scattering [14] and upconverted spontaneously parametric downconverted (SPDC) photons [15]. In this case, the upconverted SPDC photons are the only noise source overlapping with the signal frequency and it cannot be filtered away effectively. At the given spot size and power levels the effect on the SNR are, however, limited [10].

Figure 4(a) show, a comparison of the three noise contributions in the specific setup and confirm the expected low noise contributions from both the atmosphere and the upconversion process. The plot b) is included as an example of the SNR in a direct measurement of the backscattered signal from the forest.

\subsection{Angle dependency}

The change to an upconversion system results in largely improved noise specifications and collinear tests, of the upconversion module, with a $637 \mathrm{~kW} / \mathrm{cm}^{2}$ mixing field show $\mathrm{QE}>80 \%$. Still it is seen from Fig. 3(a) that the SNR is lower than with the direct InGaAs detector. For long range measurements this is a result of a decreased QE. From the theoretical description in section 2 and the configuration of signal area and angle described in section 3 the QE is estimated to be $<1 \%$. With long range applications the angular dependency is critical due to the angular spread of the signal, pointing stability issues and alignment practicalities. 

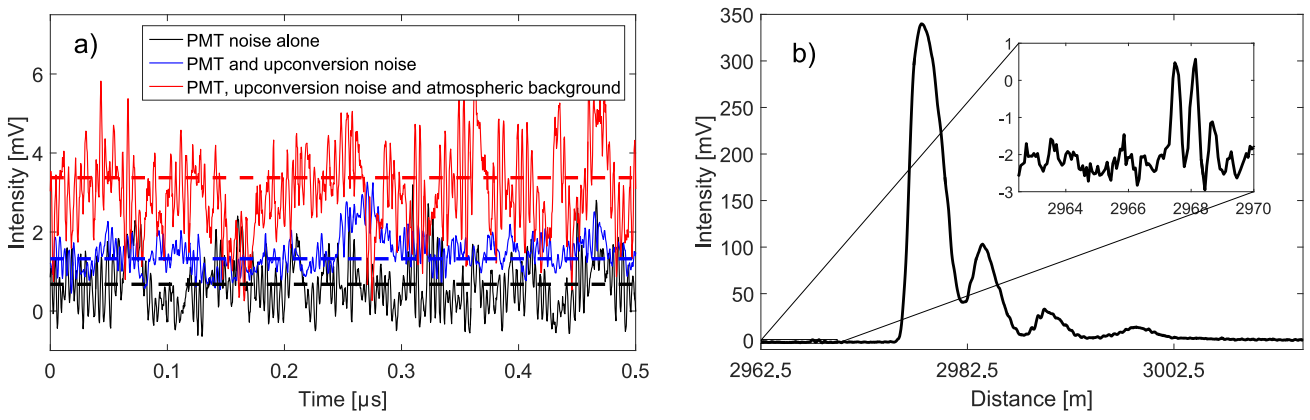

Fig. 4. a) A comparison of the different noise contributions in the combined upconversion and PMT detector. b) Example of a backscattered signal from nearby forest, illustrating the SNR for the combined PMT and upconversion detector. The oscillations after the main peak are not backscattered signal, but relaxation oscillations from the PMT. The inset show an example of the background noise. All data is an average over 32 pulse sweeps and the intensities are given as output voltages from the PMT.

To investigate the angular challenge, a measurement of the backscattering from the forest was done with a thermal gradient applied over the PPLN crystal. This is demonstrated in Fig. 5(a), where the plot show a backscattering measurement with and without the gradient applied and the peak at $2174 \mathrm{~m}$ show up uniquely on the measurement with the gradient applied. This indicates that the acceptance angle is expanded and now picks up signal from a part of the forest on a lower part of the hill closer to the receiver. The inset in Fig. 5(a) shows a thermal measurement of the crystal from above. The linear gradient at $9{ }^{\circ} \mathrm{C}$ is applied passively by replacing the brass crystal mount with one made of Teflon, designed with a thermal bridge of aluminium to the underlying heater in left end of the crystal. The gradient results in changing phasematch conditions throughout the crystal and thus expands the angle acceptance at the expense of a lower the QE. This is demonstrated both experimentally and theoretically in Fig. 5(b) where different configurations are computed and compared with acceptance angle measurements both with and without a thermal gradient. The measurements are performed by rotating the whole upconversion module in a test setup with a uniform oversize signal illumination of the PPLN
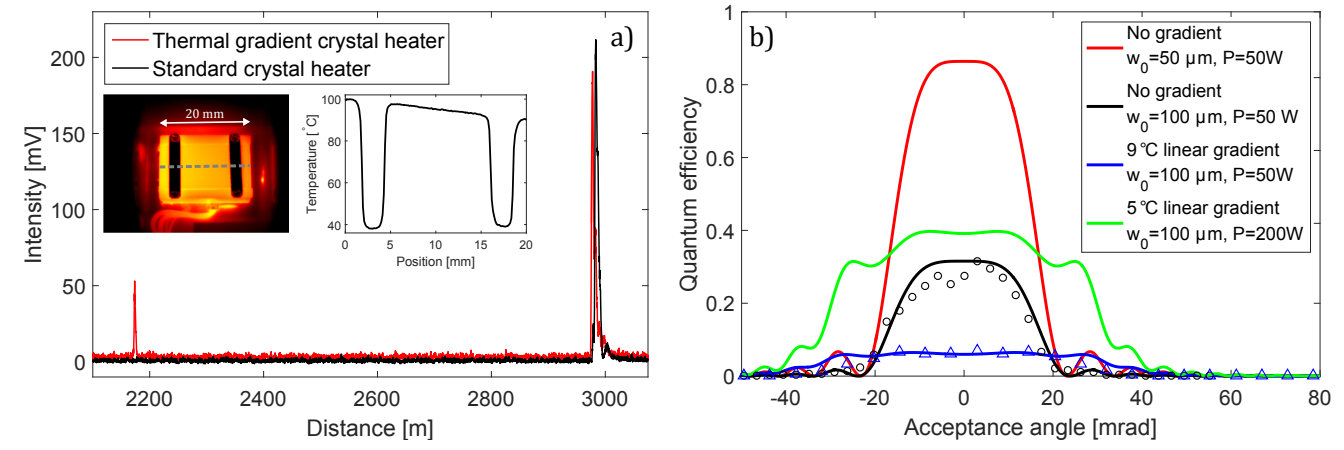

Fig. 5. a) Comparison of the backscattered signal with and without a gradient applied over the PPLN crystal. Inset show an example of a crystal with a passive gradient applied together with the corresponding surface temperatures. b) Computation of the angular acceptance for the upconversion process together with measurements normalized to fit the no gradient case. Beam waist values and mixing field powers for the simulations are included. 
channel. The upconverted power was monitored upon rotation and both measurements are calibrated equally to the computation without a gradient.

In Fig. 5(a) the consequence of a larger acceptance angle is demonstrated with the long range IPDA setup. A second peak occurs at $\sim 2180 \mathrm{~m}$, only with the thermal gradient applied, originating from trees, now within the receiver's field of view. The signal level for the main peak drops only $\sim 10 \%$, even though the $\mathrm{QE}$ for the collinear conversion is reduced by a factor of 4. Phasematch expansion in PPLN have previously been realized in [16] where the emission spectrum of a mid-IR light source was expanded through active control of a thermal gradient.

To investigate the implementation of a thermal gradient further, a plane-wave simulation based on the coupled equations for parametric interaction [8] was established. This model allows for calculations of the phasematch for different wavelengths and angles, when the temperature varies throughout the crystal. Figure 6 shows simulation results of the relation between the acceptance angle and the $\mathrm{QE}$, with respect to the influence of a linear temperature gradient, or a linear chirp of the poling structure in the crystal. The computation was done with a crystal length of $20 \mathrm{~mm}$, a poling period of $11.8 \mu \mathrm{m}$, a beam waist of $100 \mu \mathrm{m}$ and a mixing laser power of $200 \mathrm{~W}$ at $1064 \mathrm{~nm}$. This translates to a mixing field intensity on $637 \mathrm{~kW} / \mathrm{cm}^{2}$. In the case where the phasematch is optimized for collinear conversion, the angle acceptance curve will look like Fig. 5(b), with one central lobe that broadens, when a gradient is applied. In Fig. 6, the off-set temperature, at the one end of the crystal, is optimized for non-collinear interaction and the optimum is found to be $6^{\circ} \mathrm{C}$ lower than the temperature optimal for the collinear phasematch. This results in a double acceptance peak in the case without a gradient and then more regular acceptance curves for higher gradients, see Fig. 6(b). In Fig. 6(a) it is seen how the acceptance angle increases for higher gradients, but also how the average QE does not reach the optimized collinear level. The stepwise behaviour of the acceptance angle growth occurs as the phasematch at larger angles grow higher than the existing peaks at lower gradients.

The weighted efficiency, stated in Eq. 5, is plotted in Fig. 6(c) where three different angular distribution widths are treated. Each case showing an optimum gradient around $6{ }^{\circ} \mathrm{C}$. With more power confined in the small angles of the incoming field the overall QE would be higher, but as discussed earlier collinear confinement is not possible in the case of long range detection. The upper limit for the incoming angles results from the geometric bounds of a $20 \mathrm{~mm}$ long crystal with $1 \mathrm{~mm} \times 1 \mathrm{~mm}$ poling channels where angles larger than $\pm 110 \mathrm{mrad}$ will not overlap with

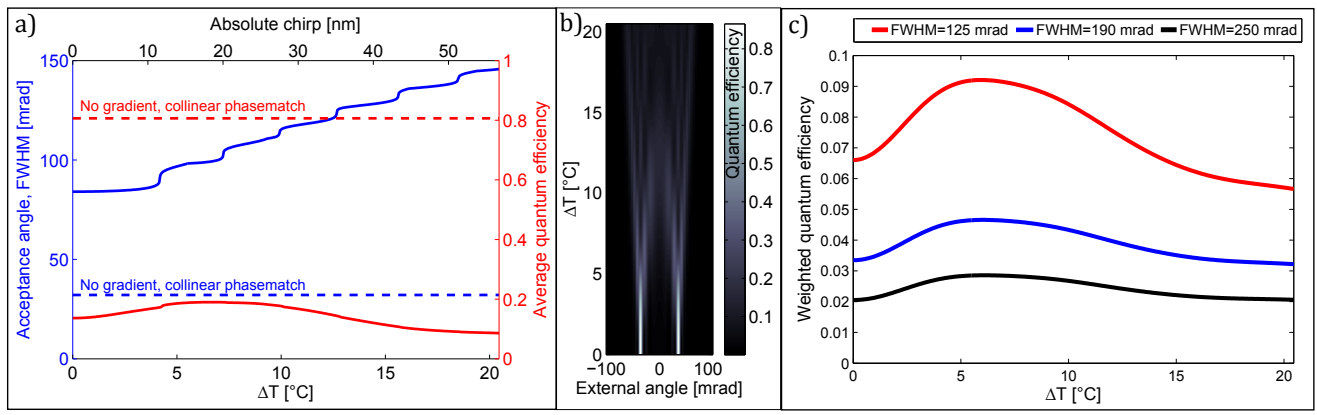

Fig. 6. a) A computation of the angular acceptance bandwidth for the upconversion process and the average QE for different values of linear temperature gradient or linear chirp of the poling pattern. b) Overview of the angular dependent QE for different temperature gradients. c) Weighted efficiency for the upconversion process calculated with respect to the temperature gradient, shown for three different angular distribution widths for the incoming signal. All plots are obtained for a mixing laser intensity of $637 \mathrm{~kW} / \mathrm{cm}^{2}$ and a off-set temperature $6^{\circ} \mathrm{C}$ below the collinear optimum. 
the poling structure for the full length of the crystal.

Chirped poling structures are an alternative to the temperature gradient and the use of such crystals is demonstrated in [17] and calculation of bandwidth expansions are presented in [18], with results that agrees well with the conclusions of our temperature gradient calculations. In Fig. 6 it is seen that an absolute chirp of approx. $30 \mathrm{~nm}$ matches a $10^{\circ} \mathrm{C}$ temperature gradient. This mean that the maximum increment from the first to the last period in the chirped structure should be $30 \mathrm{~nm}$. Such a fine chirp is not possible to resolve with present day poling procedures, and thus the temperature gradient method is preferable.

\subsection{Future improvements}

The system at hand is very experimental and built from available equipment. In an improved version of the upconversion scheme following the best case in Fig. 6(c) the mixing field is increased to $200 \mathrm{~W}$ giving 4 times higher QE or 2 times larger beam waist for the same QE, but larger overlap with the incoming signal. The visible detector could be replaced with a high QE SI-APD, or a higher QE PMT, giving a factor of 2 improvement. All these improvements would apply for both IPDA and DIAL measurements. For the DIAL measurements specifically, the detected signal would rise significantly if the transmitter beam was better collimated. The divergence can be at least four times smaller, thereby matching the receiver system better. This would give an signal in the crystal of half the size and an incoming signal with half the angular spread, increasing the overall QE by a factor of 12 . With the larger acceptance angle and higher $\mathrm{QE}$ the background noise from the atmosphere and the upconversion process could rise as well. The increased atmospheric background can be handled by a narrow band filter before and after the upconversion and the increase in noise from the upconversion process itself can be limited following the procedure for mixing intensity optimization in [10]. When all the above is taken into consideration, it is expected that the SNR of the optimized upconversion system could show an improvement of two orders of magnitude. The improved QE may allow for range resolved concentration measurements of atmospheric $\mathrm{CO}_{2}$, with much shorter integration times than demonstrated in [4]. In a future integrated implementation of the upconversion system, the beam scaling optics will be configured to match the nonlinear process. The shift in technology would only marginally increase the overall system complexity, adding a compact $14 \times 11 \mathrm{~cm}^{2}$ upconversion module and an associated power supply.

An improved upconversion system applied in a DIAL configuration shows much promise for vertical profiling of atmospheric $\mathrm{CO}_{2}$, as well as other greenhouse gases, such as methane, with slight modifications to the upconversion module.

\section{Conclusion}

It was demonstrated, for the first time, how upconversion in combination with a visible photomultiplier tube can be used for long range detection of atmospheric $\mathrm{CO}_{2}$. Measurements showed good noise properties for the combination of upconversion and visible detector technology, but also a limitation in angle acceptance and QE for the given upconversion system. With experiments and models with thermal gradient control it is analyzed how to improve the upconversion technology for long range detection.

Given the large potential in the detectivity for the visible detectors, we believe that the upconversion technology has the potential for reaching new frontiers in range resolved, long range atmospheric gas measurements. This holds true for the NIR wavelengths, and at longer wavelengths, where the discrepancy in detector noise, compared to visible detector technology, increases, the benefit of upconversion technology is even more significant [11]. 